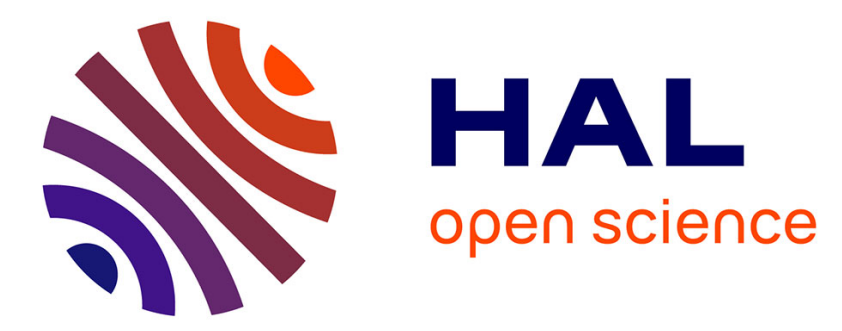

\title{
Effect of pairing on the symmetry energy and the incompressibility
}

J. Margueron, E. Khan, G. Colò, K. Hagino, H. Sagawa

\section{To cite this version:}

J. Margueron, E. Khan, G. Colò, K. Hagino, H. Sagawa. Effect of pairing on the symmetry energy and the incompressibility. European Physical Journal A, 2014, 50, pp.18. 10.1140/epja/i2014-14018-9 . in2p3-00962370

\section{HAL Id: in2p3-00962370 \\ https://hal.in2p3.fr/in2p3-00962370}

Submitted on 23 Jun 2021

HAL is a multi-disciplinary open access archive for the deposit and dissemination of scientific research documents, whether they are published or not. The documents may come from teaching and research institutions in France or abroad, or from public or private research centers.
L'archive ouverte pluridisciplinaire HAL, est destinée au dépôt et à la diffusion de documents scientifiques de niveau recherche, publiés ou non, émanant des établissements d'enseignement et de recherche français ou étrangers, des laboratoires publics ou privés.

\section{(c)(1)}

Distributed under a Creative Commons Attribution| 4.0 International License 


\title{
Effect of pairing on the symmetry energy and the incompressibility ${ }^{\star}$
}

\author{
J. Margueron ${ }^{1, a}$, E. Khan ${ }^{2}$, G. Colò ${ }^{3}$, K. Hagino ${ }^{4}$, and H. Sagawa ${ }^{5}$ \\ 1 Institut de Physique Nucléaire de Lyon, Université Claude Bernard Lyon 1, IN2P3-CNRS, F-69622 Villeurbanne, France \\ 2 Institut de Physique Nucléaire, Université Paris-Sud, IN2P3-CNRS, F-91406 Orsay Cedex, France Cedex, France \\ 3 Dipartimento di Fisica, Università degli Studi and INFN, Sezione di Milano, Via Celoria 16, 20133 Milano, Italy \\ 4 Department of Physics, Tohoku University, Sendai, 980-8578, Japan \\ ${ }^{5}$ Center for Mathematics and Physics, University of Aizu, Aizu-Wakamatsu, 965-8580 Fukushima, Japan
}

\begin{abstract}
The role of superfluidity on the symmetry energy and on the incompressibility is studied in nuclear matter and finite nuclei employing Hartree-Fock-Bogoliubov modeling based on several types of pairing interactions (surface, mixed and isovectordensity dependent). It is observed that, while pairing has only a marginal effect on the symmetry energy and on the incompressibility at saturation density, the effects are significantly larger at lower densities.
\end{abstract}

\section{Introduction}

The nuclear symmetry energy and the incompressibility are closely related to the isovector Giant Dipole Resonance (GDR) [1] and to the isoscalar Giant Monopole Resonance (GMR) $[2,3]$, respectively. The symmetry energy, and in particular its density dependence, as well as the isospin dependence of the incompressibility modulus are largely debated issues in nuclear physics at present. In fact, these issues have relevant implications i) for nuclear structure, since the symmetry energy has an important effect on the size of the neutron root-mean-square (r.m.s.) radius in neutron-rich nuclei and the incompressibility is related to the GMR centroid, ii) for nuclear reactions, e.g., in intermediate energy heavy-ion collisions where the isospin distribution of the reaction products is dictated by the density dependence of the symmetry energy, and obviously iii) for the description of neutron stars and their formation in core-collapse supernovae. Review papers have been devoted to this topic $[4,5]$. Empirical information on the symmetry energy can be obtained from various sources, none of them being so far conclusive by itself. Measurements of the neutron skin, in lead, for instance, are still not conclusive enough: while most of them are plagued by unknown model dependence, the recent model-independent PREX measurement [6] could not yet reach the promised accuracy. The properties of

\footnotetext{
* Contribution to the Topical Issue "Nuclear Symmetry Energy" edited by Bao-An Li, Àngels Ramos, Giuseppe Verde, Isaac Vidaña.

a e-mail: jmargue@ipno.in2p3.fr
}

the isovector GDR, of the low-lying electric dipole excitations, and of the charge-exchange spin-dipole strength have been suggested as constraints (see, e.g., [7]). In addition, different model analysis of heavy-ion collisions have been proposed as a test of the main trend of the symmetry energy at densities below saturation. However, in none of these studies, to our knowledge, the problem of the pairing effects on the symmetry energy has been addressed.

The apparent decrease of incompressibility in superfluid nuclei $[8,9]$ raises the question about a possible similar effect in infinite nuclear matter: until now, when the nuclear incompressibility is extracted from Energy Density Functional (EDF) calculations of the GMR, and compared with experiments, there has been no attempt to pin down the contribution of the pairing component of the functional. However, considering results for finite nuclei, the equations of state used for neutron stars and supernovae predictions should take into account pairing effects in the calculation of the incompressibility modulus. Therefore the question of the behavior of $K_{\infty}$ with respect to the pairing gap is raised since it seems clear from nuclear data that the finite nucleus incompressibility $K_{A}$ decreases with increasing pairing gap [8]. A similar study for nuclear matter, as well as a more systematic study in finite nuclei, should be undertaken. This is the goal of the present work [10]. It should be also noted that we will not consider the neutron-proton $T=0$ pairing channel since the nuclei considered are far from $N=Z$.

In this paper, the effects of the pairing correlations on the symmetry energy and on the incompressibility are studied consistently in nuclear matter and in finite nuclei. The effects coming from the correlation energy associated 
with the pairing force are included. It should be noted that the surface versus the mixed nature of the pairing interaction is still under discussion. For instance, a recent systematic study based on the odd-even mass staggering seems to slightly favor a surface type of pairing interaction [11]. In the following, we will therefore explore various kinds of pairing interactions.

\section{Nuclear matter}

In this section, we study the effects of the pairing correlations on the symmetry energy and incompressibility in nuclear matter.

\subsection{Energy density}

The nuclear energy density $\epsilon(\epsilon=E / V)$ is the sum of the Skyrme part, $\epsilon_{\text {Skyrme }}$, that includes the kinetic energy [12], plus the pairing energy density,

$$
\epsilon=\epsilon_{\text {Skyrme }}+\epsilon_{\text {pair }}
$$

Here

$$
\epsilon_{\mathrm{pair}}=-\frac{1}{2}\left(N_{n} \Delta_{n}^{2}+N_{p} \Delta_{p}^{2}\right) .
$$

In eq. (2), $\Delta_{\tau}$ is the pairing gap and $N_{\tau}$ is the density of states, given by $N_{\tau}=m_{\tau}^{*} k_{F \tau} /\left(2 \pi^{2} \hbar^{2}\right)$, with $\tau=n, p$. The energy density $\epsilon$ is a function of the total density $\rho$ and of the asymmetry parameter $\delta=\left(\rho_{n}-\rho_{p}\right) / \rho$. In the $T=1$ channel, several pairing interactions are defined by

$$
v_{\text {pair }}^{\text {IS }}\left(\boldsymbol{r}, \boldsymbol{r}^{\prime}\right)=v_{0}\left(1-\eta\left(\frac{\rho}{\rho_{0}}\right)^{\alpha}\right) \delta\left(\boldsymbol{r}-\boldsymbol{r}^{\prime}\right),
$$

as a function of the value of $\eta$ that can range from 0 (volume-type pairing) to 1 (surface-type pairing). In eq. (3) the parameter $\alpha$ is set to 1 and $\rho_{0}$ is taken as the saturation density of symmetric nuclear matter throughout all the study; moreover, we adopt the parameters $\eta=0.35$ and 0.65 for the volume-surface mixed-type pairing interactions, and $\eta=1.0$ for the surface-type interaction. The values of $v_{0}$ in all these cases are adjusted, for each $\eta$, in such a way to obtain equivalent results for the two neutron separation energy in the Sn isotopes by Hartree-Fock-Bogoliubov (HFB) calculations with the SLy5 parameter set [12]. The pairing cutoff energy is set at $60 \mathrm{MeV}$ [13]. These values of $v_{0}$ are given in table I of ref. [10]. In the following, these pairing interactions will be denoted as IS, because they depend on the isoscalar density.

We have also considered pairing interactions having the isovector density dependence with $\delta=\left(\rho_{n}-\rho_{p}\right) / \rho$ in addition to the isoscalar density dependence, defined either as [14]

$$
\begin{aligned}
v_{\text {pair }}^{\mathrm{MSH}}\left(\boldsymbol{r}, \boldsymbol{r}^{\prime}\right)= & v_{0}\left[1-(1-\delta) \eta_{s}\left(\frac{\rho}{\rho_{0}}\right)^{\alpha_{s}}\right. \\
& \left.-\delta \eta_{n}\left(\frac{\rho}{\rho_{0}}\right)^{\alpha_{n}}\right] \delta\left(\boldsymbol{r}-\boldsymbol{r}^{\prime}\right),
\end{aligned}
$$

with $v_{0}=-448 \mathrm{MeV} \cdot \mathrm{fm}^{3}, \eta_{s}=0.598, \alpha_{s}=0.551, \eta_{n}=$ $0.947, \alpha_{n}=0.554$ (with a cutoff energy of $60 \mathrm{MeV}$ ), or as $[15]$

$$
\begin{aligned}
v_{\text {pair }}^{\mathrm{YS}}\left(\boldsymbol{r}, \boldsymbol{r}^{\prime}\right)= & v_{0}\left[1-\left(\eta_{0}+\eta_{1} \tau_{3} \delta\right) \frac{\rho}{\rho_{0}}\right. \\
& \left.-\eta_{2}\left(\delta \frac{\rho}{\rho_{0}}\right)^{2}\right] \delta\left(\boldsymbol{r}-\boldsymbol{r}^{\prime}\right),
\end{aligned}
$$

with $v_{0}=-344 \mathrm{MeV} \cdot \mathrm{fm}^{3}, \eta_{0}=0.5, \eta_{1}=0.2, \eta_{2}=2.5$ (with a cutoff energy of $50 \mathrm{MeV}$ ). The parameters mentioned have been used in connection with the SLy5 interaction. In the following these pairing interactions will be denoted as IS+IV.

The pairing gap in uniform matter is obtained from the BCS gap equation [16]

$$
\Delta_{k}=\sum_{k^{\prime}} v_{k k^{\prime}} \frac{\Delta_{k^{\prime}}}{E_{k^{\prime}}},
$$

solved under the condition of the particle number conservation. In a given volume $V$ one assumes constant density given by

$$
\rho_{\tau}=\frac{2}{V} \sum_{k}\left(1-\frac{e_{\tau}(k)-\mu_{\tau}}{E_{k, \tau}}\right),
$$

where the quasiparticle energy is defined as

$$
E_{k, \tau}=\overline{\left(e_{\tau}(k)-\mu_{\tau}\right)^{2}+\Delta_{k, \tau}^{2}},
$$

$e_{\tau}(k)$ being the single-particle energy, and $\mu_{\tau}$ is the chemical potential. In eq. (6), $v_{k k^{\prime}}$ is the pairing matrix element for the plane waves, namely $\left\langle k \bar{k}|v| k^{\prime} \bar{k}^{\prime}\right\rangle$. Notice that in the case of the zero-range pairing interaction, the pairing gap $\Delta_{k}$ is independent of $k$.

In fig. 1 we display the pairing gap $\Delta_{\tau}$, the pairing energy per particle $e_{\text {pair }}$ and the percentage of the pairing energy with respect to the total energy $e$ in symmetric matter for the various pairing interactions together with the SLy5 Skyrme interaction [12] in the mean-field channel. There is a critical density $\rho_{c} \approx 0.11 \mathrm{fm}^{-3}$ at which the pairing interactions, which have been adjusted on nuclear energies (IS 0.35 , IS 0.65 , IS 1.0, YS), give almost the same result for the pairing gap, around $1.5 \mathrm{MeV}$. This has already been noticed in ref. [13] and may be related to the fact that, in fitting the two-neutron separation energy, one is sensitive to the space region of the nuclear surface, where the density is somewhat lower than the saturation density: therefore the pairing gap is constrained rather at $\rho_{c}$ than at $\rho_{0}$. Above $\rho_{c}$, the more surface-type the pairing interaction (that is, the larger $\eta$ is taken), the smaller the pairing gap $\Delta_{\tau}$. Below the critical density, the trend is reversed: the more surface-type the pairing interaction is, the larger the pairing gap. The contribution of the pairing energy is increased at low densities. Around the saturation density, the pairing energy per particle is much smaller than the binding energy $(-16 \mathrm{MeV})$.

In fig. 2 we display the pairing gaps $\Delta_{n}$ and $\Delta_{p}$, the pairing energy per particle $e_{\text {pair }}$ and the total energy versus the asymmetry parameter $\delta=(N-Z) /(N+Z)$ 

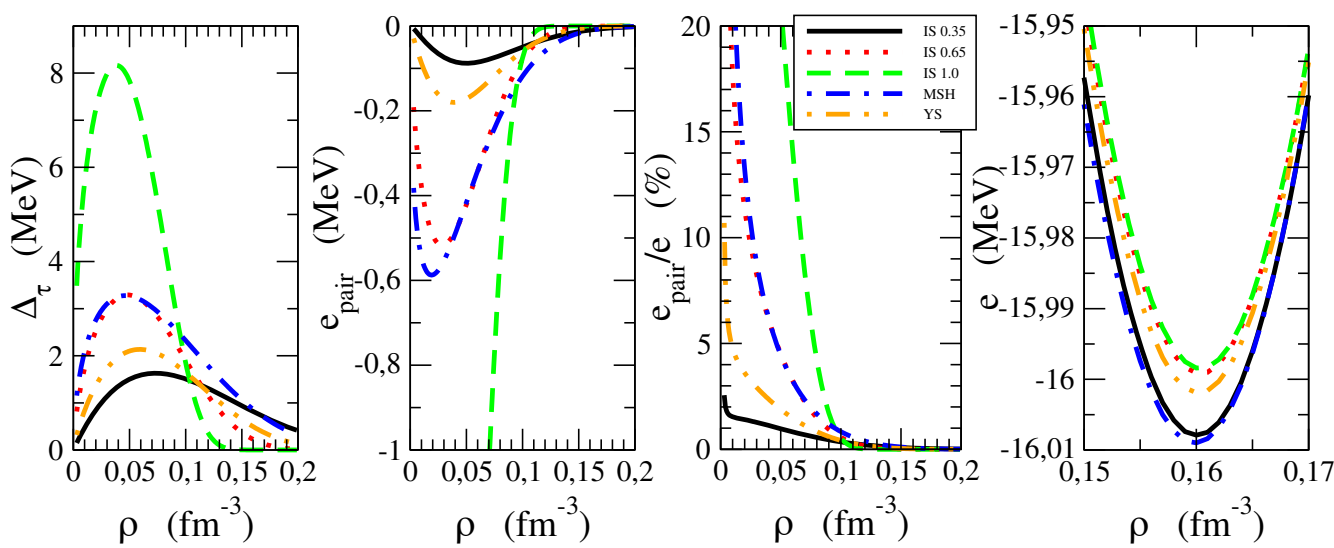

Fig. 1. Pairing gap $\Delta_{\tau}$, pairing energy per particle $e_{\text {pair }}$, percentage of pairing energy with respect to the total energy $e$ and equation of state around the saturation point in symmetric matter obtained with various pairing interactions employed in connection with the SLy5 Skyrme interaction. The solid, dotted and dashed lines correspond to the IS pairing interactions with $\eta=0.35,0.65$ and 1.0, respectively, in eq. (3). The dash-dotted and dash-dot-dotted lines show the results of IS+IV interactions in ref. [15] (MSH) and ref. [16] (YS), respectively. See the text for details.

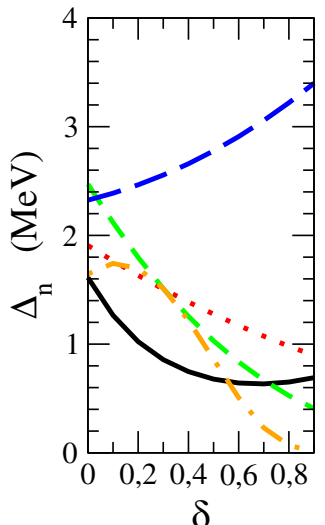

$\delta$

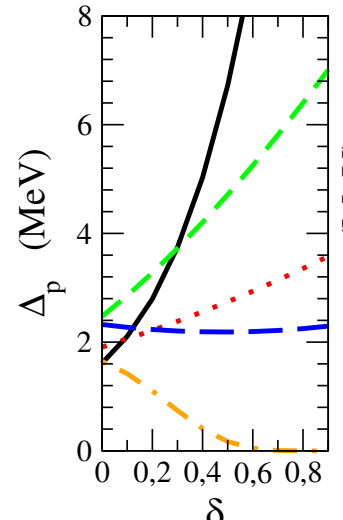

$\delta$
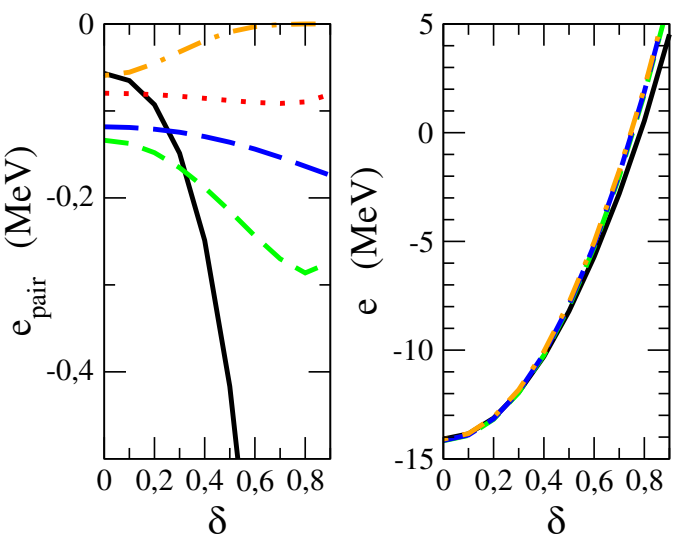

Fig. 2. Pairing gaps $\Delta_{n}$ and $\Delta_{p}$, pairing energy per particle $e_{\text {pair }}$, and total energy per particle $e$, in asymmetric nuclear matter characterized by the asymmetry $\delta=(N-Z) /(N+Z)$ and where the density is fixed to be $0.1 \mathrm{fm}^{-3}$. We used SLy5 Skyrme interaction and the different pairing forces are the same as in fig. 1.

for the total density fixed to the average density in nuclei $\left(\sim 0.11 \mathrm{fm}^{-3}\right)$. It is observed a systematic decrease (respectively, increase) of the pairing gap $\Delta_{n}$ (respectively, $\Delta_{p}$ ) as the asymmetry parameter increases, for the isoscalar pairing interactions (IS 0.35, IS 0.65 and IS 1.0). This phenomenon is not related to the pairing interaction itself, since for a fixed total density, the pairing interaction is contant, see eq. (3). This phenomenon is therefore uniquely related to the single-particle spectrum in asymmetric matter, and in particular, to the asymmetry dependence of the effective mass. The two other interactions MSH and YS are function of the isospin asymmetry, and therefore the asymmetry dependence of the pairing gap depends both on the interaction itself and on the single-particle spectrum in asymmetric matter. The pairing interaction MSH, been adjusted in the microscopic BCS pairing gaps in symmetric and neutron matter, show the expectations from microscopic calculation for both $\delta=0$ and 1 . Let us notice that there is a large spreading around these expectations depending on the pairing interactions.

\subsection{Symmetry energy and incompressibility}

The density-dependent symmetry energy $S(\rho)$ is defined by

$$
S(\rho)=\left.\frac{1}{2} \frac{\partial^{2} \epsilon / \rho}{\partial \delta^{2}}\right|_{\delta=0},
$$

and it can be expanded, around the saturation density, as

$$
S(\rho)=J+L\left(\frac{\rho-\rho_{0}}{3 \rho_{0}}\right)+\frac{1}{2} K_{\mathrm{sym}} \delta^{2}\left(\frac{\rho-\rho_{0}}{3 \rho_{0}}\right)^{2},
$$

where $J$ is defined by $J=S\left(\rho_{0}\right), L=\left.\frac{3}{\rho_{0}} \frac{\partial S}{\partial \rho}\right|_{\rho_{0}}$, and $K_{\text {sym }}=\left.\frac{9}{\rho_{0}^{2}} \frac{\partial^{2} S}{\partial \rho^{2}}\right|_{\rho_{0}}$.

We can define the density-dependent incompressibility as $[17,10]$

$$
K(\rho)=9 \rho^{2} \frac{\partial^{2} E / A}{\partial \rho^{2}}+\frac{18}{\rho} P,
$$

which coincides with the incompressibility $K_{\infty}=$ $9 \rho_{0}^{2} \frac{\partial^{2} E / A}{\partial \rho^{2}}$ at the saturation density. 

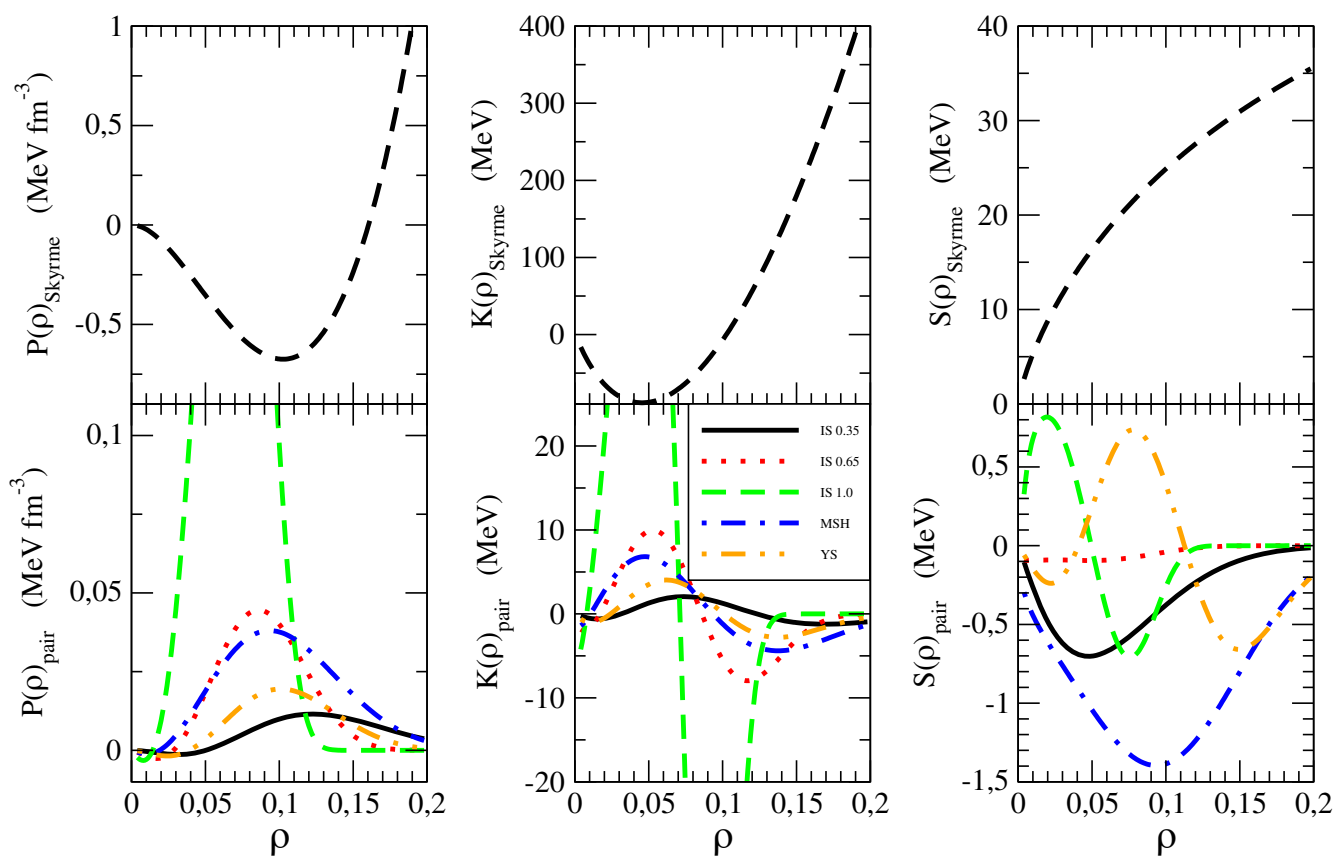

Fig. 3. Pressure, incompressibility, and symmetry energy without pairing (top panels), and the contributions of various pairing interactions to these quantities (bottom panels). The Hartree-Fock (HF) energy $\epsilon_{\text {Skyrme }}$ is calculated using the SLy5 interaction. For details, see the caption to fig. 1 and the text.

Table 1. Properties of nuclear matter for various pairing interactions. The SLy5 Skyrme force is used for the mean field.

\begin{tabular}{ccccccc}
\hline \hline Pairing & $\begin{array}{c}\rho_{0} \\
{\left[\mathrm{fm}^{-3}\right]}\end{array}$ & $\begin{array}{c}E / A\left(\rho_{0}\right) \\
{[\mathrm{MeV}]}\end{array}$ & $\begin{array}{c}K_{\infty} \\
{[\mathrm{MeV}]}\end{array}$ & $\begin{array}{c}J \\
{[\mathrm{MeV}]}\end{array}$ & $\begin{array}{c}L \\
{[\mathrm{MeV}]}\end{array}$ & $\begin{array}{c}K_{\text {sym }} \\
{[\mathrm{MeV}]}\end{array}$ \\
\hline \hline no pairing & 0.1604 & -15.999 & 230.2 & 32.03 & 48.25 & -112.3 \\
\hline IS $\eta=0.35$ & 0.1601 & -15.998 & 227.3 & 31.93 & 48.49 & -129.7 \\
IS $\eta=0.65$ & 0.1603 & -15.998 & 228.1 & 32.02 & 48.30 & -113.7 \\
IS $\eta=1.00$ & 0.1604 & -15.999 & 230.1 & 32.03 & 48.25 & -112.3 \\
MSH & 0.1599 & -15.998 & 223.9 & 31.33 & 55.77 & -139.7 \\
YS & 0.1602 & -15.998 & 227.0 & 31.39 & 52.04 & 13.2 \\
\hline \hline
\end{tabular}

\subsection{Results}

Figure 3 displays the pressure $P$, the incompressibility $K(\rho)$ in eq. (11), and the symmetry energy $S(\rho)$ in eq. (10) without pairing (top panels), and the contribution of pairing to these quantities (bottom panels), using the SLy5 interaction [12]. This contribution is calculated with the same equations, but considering only the pairing term of the energy density in fig. 1 . The same pairing interactions have been considered here as in fig. 1. Close to the saturation density, the contribution from pairing is very small. This is also illustrated in table 1: the pairing interaction has small effects at the saturation density. In the case of the incompressibility $K_{\infty}$, pairing can still produce a few $\%$ effect (for instance, $K_{\infty}$ is changed from $230.2 \mathrm{MeV}$ to $223.9 \mathrm{MeV}$ in the case of the MSH pairing interaction).
The MSH, YS, IS 0.35 pairing interactions modify the incompressibility by 3 to $6 \mathrm{MeV}$, that is, by about $2 \%$. It should be noted that at the saturation density, the contribution to the slope parameters of the symmetry energy, $L$, and $K_{\text {sym }}$, of the interactions $\mathrm{MSH}$ and $\mathrm{YS}$ is larger than that of the other IS forces. The effects on $L$ can be about $15 \%$ while $K_{\text {sym }}$ can be modified in an important way. This is related to the dependence of these pairing interaction on the isovector density.

However at lower densities, the pairing effects become appreciably larger as seen in fig. 3. In the case of the pure surface pairing, there are important contributions to the pressure, incompressibility and symmetry energy: these quantities can be strongly affected by pairing, which can lead to variations up to about a factor 2. Other pairing interactions also provide significant corrections to the pressure and the incompressibility, typically, around $10 \%$. In the case of the symmetry energy, for typical densities $\rho \approx 0.1 \mathrm{fm}^{-3}$, the IS+IV pairing interaction YS predict a positive contribution which is opposite to all the other interactions considered here. It should be noted that the pairing contribution to these quantities is generally larger at densities below saturation.

To obtain a more general view of the pairing effect on the incompressibility, table 2 displays the $K_{\infty}$ values obtained for SLy5, LNS, Sk255 and Sk272 Skyrme functionals, with various pairing interactions. In table 2 , the pairing interaction IS 0.35 is the largest one among the IS interactions and reduces the incompressibility $K_{\infty}$ by about $3 \mathrm{MeV}$. The MSH interaction induces a correction of $6.3 \mathrm{MeV}$ on the incompressibility. It should be noted that the pure surface pairing interaction provides no modification of $K_{\infty}$. Depending on the Skyrme models, there 
Table 2. Nuclear matter incompressibility $K_{\infty}(\mathrm{MeV})$ for SLy5 [12], LNS [18], Sk255 [19] and Sk272 [19] Skyrme functionals. The dependence of $K_{\infty}$ on the pairing interaction is displayed: mixed (IS $\eta=0.35$ ), surface (IS $\eta=1.00$ ). The effect of the MSH pairing is also displayed in the SLy5 case.

\begin{tabular}{ccccc}
\hline \hline Pairing & SLy5 & LNS & Sk255 & Sk272 \\
\hline \hline no pairing & 230.2 & 211.0 & 255.2 & 271.8 \\
\hline IS $\eta=0.35$ & 227.3 & 208.4 & 251.3 & 268.3 \\
IS $\eta=1.00$ & 230.1 & 211.0 & 255.2 & 271.8 \\
MSH & 223.9 & - & - & - \\
\hline \hline
\end{tabular}

shall also be an effect due to the different effective masses $m^{*} / m$, but they are incorporated in the renormalization of the pairing interaction parameter $v_{0}$.

It is expected that the above pairing effects at low densities may also affect finite nuclei. In the case of incompressibility, we can define a finite nucleus value $K_{A}$ and expect that this value is affected by the pairing more than $K_{\infty}$, due to the presence of a lower density region, i.e. the nuclear surface. We analyze this point in the next section, and we argue that a similar reasoning holds for the symmetry energy.

\section{Local density approximation (LDA)}

This section relates the general expressions in uniform matter obtained in sect. 2 with the observables in finite nuclei in the local density approximation. The aim is to estimate the role of pairing in the incompressibility and symmetry energy of finite nuclei in a simple and transparent way. The validity of the LDA will be estimated by comparing the predicted nuclei incompressibility with the one obtained by a microscopic approach.

The binding energy per nucleon in the LDA reads

$$
B_{A}(N, Z)=B_{\text {Nucl. }}(N, Z)+B_{\text {Coul. }} \frac{Z^{2}}{A^{4 / 3}},
$$

where $B_{\text {Nucl. }}(N, Z)$ includes the bulk, surface and pairing contributions. It is defined by

$$
B_{\text {Nucl. }}(N, Z)=\frac{1}{A} \int \mathrm{d}^{3} r \epsilon(r),
$$

where $\epsilon(r)=\epsilon\left(\rho_{n}(r), \rho_{p}(r)\right)=\epsilon_{\text {Skyrme }}(r)+\epsilon_{\text {pair }}(r)$ as was defined in eq. (1). The neutron and proton densities $\rho_{n}(r), \rho_{p}(r)$ can be obtained, in the present context, by means of a spherical HF calculation. The pairing contribution to the binding energy is defined by

$$
B_{\text {pair }}(N, Z)=\frac{1}{A} \int \mathrm{d}^{3} r \epsilon_{\text {pair }}(r) .
$$

$B_{\text {Nucl. }}$ can be expanded around the saturation density,

$$
B_{\mathrm{Nucl} .}(N, Z) \approx B_{\infty}+\frac{1}{2} K_{A}\left(\frac{\rho-\rho_{0}}{3 \rho_{0}}\right)^{2}+S_{A} \delta^{2},
$$

where the symmetry energy in nuclei, $S_{A}$, is defined by

$$
S_{A}=\left.\frac{1}{2} \frac{\partial^{2} B_{\mathrm{Nucl} .}(N, Z)}{\partial \delta^{2}}\right|_{\delta=0}
$$

and the contribution of the pairing correlations to $S_{A}$ is defined by

$$
S_{\text {pair }}=\left.\frac{1}{2} \frac{\partial^{2} B_{\text {pair }}(N, Z)}{\partial \delta^{2}}\right|_{\delta=0}
$$

The incompressibility in nuclei, $K_{A}$, is defined by $K_{A}=K_{\text {Nucl. }}+K_{\text {Coul. }} \cdot Z^{2} A^{-4 / 3}$, and

$$
K_{\text {Nucl. }}=9 \rho_{0}^{2} \frac{\partial^{2} B_{\text {Nucl. }}(N, Z)}{\partial \rho^{2}},
$$

while the pairing contribution to the incompressibility is defined by

$$
K_{\text {pair }}=9 \rho_{0}^{2} \frac{\partial^{2} B_{\text {pair }}(N, Z)}{\partial \rho^{2}} .
$$

The Coulomb contribution, $K_{\text {Coul. }}$, can be evaluated using, for instance, the Thomas-Fermi approximation (cf. eq. (A1) in ref. [20]). It will not be included in the present work but the value obtained in ref. [20] is $-8 \mathrm{MeV}$ $<K_{\text {Coul. }}<-4 \mathrm{MeV}$, depending on the interaction which is used.

Introducing the mass formula (13) into eq. (18), one obtains

$$
K_{\mathrm{Nucl} .}=\frac{\rho_{0}}{A} \int \mathrm{d}^{3} r K_{\mathrm{Nucl} .}(r)
$$

with

$$
K_{\text {Nucl. }}(r)=\frac{\rho_{0}}{\rho} K(\rho(r)) .
$$

For small values of the density $\left(\rho<0.6 \rho_{0}\right.$, that is $r>5 \mathrm{fm}$ in ${ }^{120} \mathrm{Sn}$ ), the incompressibility is found to be negative: this is due to the spinodal instability in nuclear matter which is not present in finite systems [21]. For this reason, the integral (20) is limited to the region where $K_{\text {Nucl. }}(r)$ is positive. In this way, the spurious component due to the spinodal instability is removed.

Introducing the quantity

$$
S_{A}(r)=\left.\frac{1}{2 \rho_{0}} \frac{\partial^{2} \epsilon}{\partial \delta^{2}}\right|_{\delta=0}=\frac{\rho}{\rho_{0}} S(\rho(r)),
$$

the symmetry energy in nuclei (16) reads

$$
S_{A}=\frac{\rho_{0}}{A} \int \mathrm{d}^{3} r S_{A}(r) .
$$

We first perform a self-consistent $\mathrm{HF}$ calculation which provides the neutron and proton densities in ${ }^{120} \mathrm{Sn}$. From these densities we deduce the radial distributions of meanfield part and pairing part of $\epsilon(r)$ given in eq. (1), $K_{\text {Nucl. }}(r)$ given in eq. (21), and $S_{A}(r)$ given in eq. (22): these radial functions are shown in fig. 4. As expected from the results discussed in the previous section, the pairing effects on $\epsilon(r), K_{\text {Nucl. }}(r)$ and $S_{A}(r)$ come from the low-density surface region. 

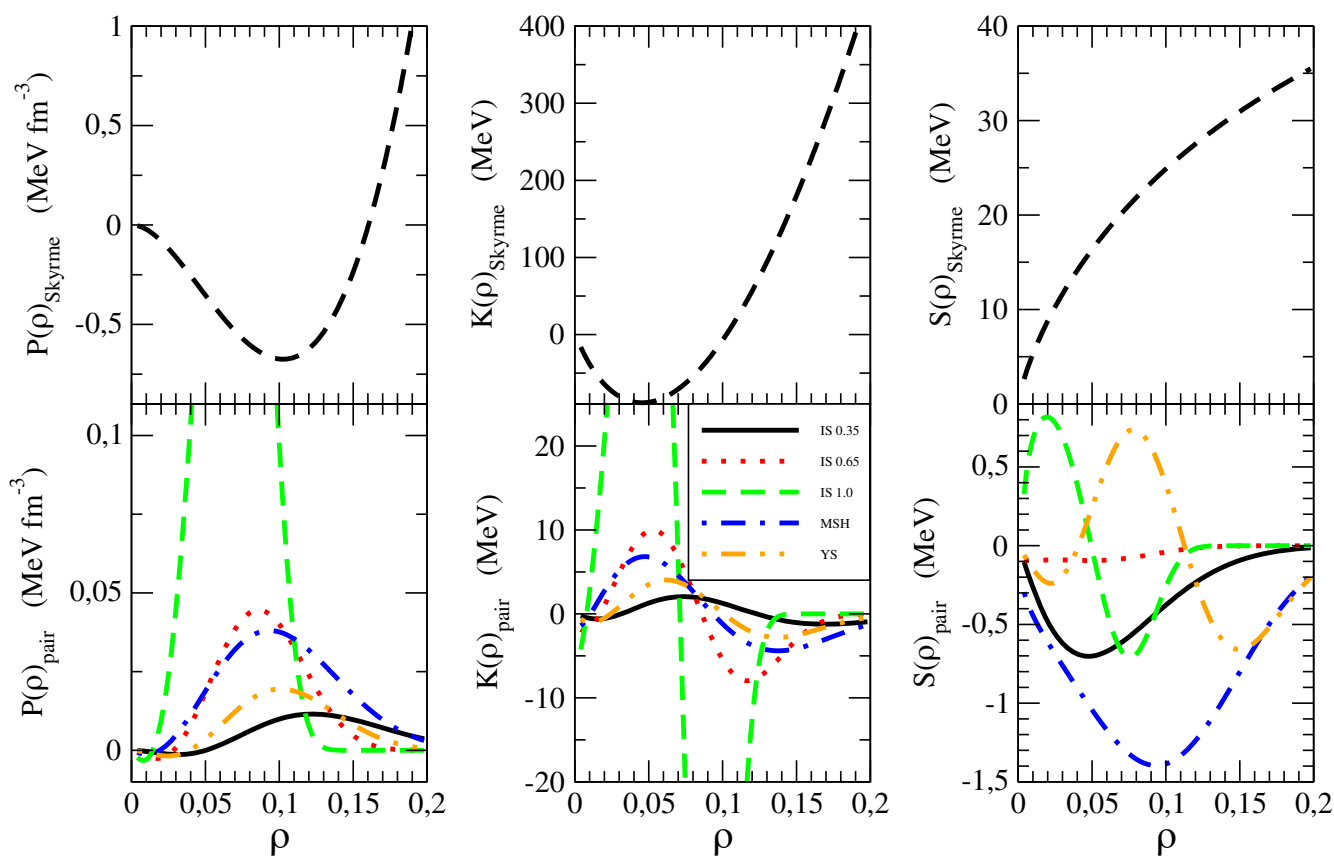

Fig. 4. Radial dependence of $\epsilon(r), K_{\text {Nucl. }}(r)$ and $S_{A}(r)$ for the various pairing interactions considered (bottom panel) using the SLy5 force in ${ }^{120} \mathrm{Sn}$ within the LDA. The contribution of the Skyrme term plus the kinetic term is displayed in the top panel. See the caption of fig. 1 and the text for details.

Table 3. Contributions of pairing correlations to the binding energy, the incompressibility and the symmetry energy in ${ }^{120} \mathrm{Sn}$. The mean field is calculated by using SLy5 interaction

\begin{tabular}{cccc}
\hline \hline Pairing & $\begin{array}{c}B_{\text {pair }} \\
{[\mathrm{MeV}]}\end{array}$ & $\begin{array}{c}K_{\text {pair }} \\
{[\mathrm{MeV}]}\end{array}$ & $\begin{array}{c}S_{\text {pair }} \\
{[\mathrm{MeV}]}\end{array}$ \\
\hline IS $\eta=0.35$ & -0.03 & -0.5 & -0.25 \\
IS $\eta=0.65$ & -0.11 & -3.9 & -0.03 \\
IS $\eta=1.00$ & -0.64 & -6.0 & -0.03 \\
MSH & -0.13 & -3.2 & -0.93 \\
YS & -0.05 & -1.9 & -0.24 \\
\hline \hline
\end{tabular}

From eqs. (13), (20) and (23), we obtain, in the SLy5 case, $B_{A}=-13.5 \mathrm{MeV}, K_{\text {Nucl. }}=119.8 \mathrm{MeV}$, and $S_{A}=$ $25.7 \mathrm{MeV}$ without the contribution due to the pairing correlations. The Coulomb contribution has not been included. The value for $K_{\text {Nucl. }}$ should be compared with that of $141 \mathrm{MeV}$ obtained by the constrained HFB (CHFB) calculations presented in ref. [10]. It should be noted that the CHFB calculations take into account the contribution coming from the Coulomb interaction. This contribution is estimated to be about $20 \mathrm{MeV}$ in ${ }^{120} \mathrm{Sn}$, using the values of $K_{\text {Coul. }}$ from ref. [20]. The good agreement between the LDA and the CHFB results ensures that LDA provides a sound framework to relate the nuclear matter incompressibility and the finite nucleus one.

The contributions of pairing correlations to the binding energy, the bulk modulus and the symmetry energy are shown in table 3 for the various pairing interactions considered. The contribution of the surface-type pairing (IS $\eta=1.0$ ) reduces $K_{A}$ by about $5 \%$, whereas, for the IS mixed-type $(\eta=0.35$ or 0.65$)$ and the IS+IV (MSH and YS) pairing interactions, the effect on $K_{A}$ is predicted to be smaller. In table 3 , it is also observed that pairing effects affect the binding energy by few percents, up to $5 \%$ for the surface-type pairing interaction. For the symmetry energy, pairing effects are negligible, being below $1 \%$ except the IS+IV pairing (MSH).

\section{Conclusions}

The effect of superfluidity on the symmetry energy and the incompressibility has been studied in both nuclear matter and finite nuclei, using various pairing energy density functionals. A small effect is observed on the nuclear matter incompressibility and the volume symmetry energy at the saturation point, but the effect is non-negligible on the derivative terms, $L$ and $K_{\mathrm{sym}}$, especially in the case of IS+IV pairing.

However at lower density, the pairing effect on the incompressibility is significant and can have a substantial impact on neutron stars studies or on the interpretation of multifragmentation data. It has been shown that the LDA provides a relevant framework for a qualitative understanding and interpretation of the microscopic results. The effect of the pairing correlations is localized near the surface of nuclei and the effect of the pairing correlations is to make slightly softer nuclear EOS. Especially in the low-density region in nuclear matter, the pairing effect is more noticeable. This may explain why such effects are expected to happen in the surface of the finite nuclei. In 
the case of the IS+IV pairing interaction, no strong effect is observed on $K_{A}$. In general, the pairing effects on the finite nucleus incompressibility $K_{A}$ are more important when the interaction is more surface type (larger $\eta$ value).

This study shows that with respect to current experimental uncertainties, the pairing effects should be considered when extracting the incompressibility value from GMR data which can now reach an accuracy of several hundreds of $\mathrm{keV}$ [22]. Experimentally it would be useful to measure the GMR on isotopic chains, including both open-shell and doubly magic nuclei such as ${ }^{132} \mathrm{Sn}$. Such measurements are starting to be undertaken [22-24] and will be extended to unstable nuclei [25].

This work was supported by the Japanese Ministry of Education, Culture, Sports, Science and Technology by Grant-in-Aid for Scientific Research under the program numbers 20540277 and 22540262, and by the ANR SN2NS contract.

\section{References}

1. L. Trippa, G. Colò, E. Vigezzi, Phys. Rev. C 77, 061304(R) (2008).

2. J.-P. Blaizot, Phys. Rep. 64, 171 (1980).

3. G. Colò, N. Van Giai, J. Meyer, K. Bennaceur, P. Bonche, Phys. Rev. C 70, 024307 (2004).

4. A.W. Steiner, M. Prakash, J.M. Lattimer, P.J. Ellis, Phys. Rep. 411, 325 (2005).

5. B.A. Li, Lie-Wen Chen, Che Ming Ko, Phys. Rep. 464 113 (2008).
6. S. Abrahamyan et al., Phys. Rev. Lett. 108, 112502 (2012).

7. A. Klimkiewicz et al., Phys. Rev. C 81, 041301 (2010).

8. E. Khan, Phys. Rev. C 80, 011307(R) (2009).

9. E. Khan, Phys. Rev. C 80, 057302 (2009).

10. E. Khan et al., Phys. Rev. C 82, 024322 (2010).

11. G.F. Bertsch, C.A. Bertulani, W. Nazarewicz, N. Schunck, M.V. Stoitsov, Phys. Rev. C 79, 034306 (2009).

12. E. Chabanat, P. Bonche, P. Haensel, J. Meyer, R. Schaeffer, Nucl. Phys. A 635, 231 (1998).

13. E. Khan, M. Grasso, J. Margueron, Phys. Rev. C 80, 044328 (2009).

14. J. Margueron, H. Sagawa, K. Hagino, Phys. Rev. C 76, 064316 (2007).

15. M. Yamagami, Y.R. Shimizu, Phys. Rev. C 77, 064319 (2008).

16. J. Bardeen, L.N. Cooper, J.R. Schrieffer, Phys. Rev. 108, 1175 (1957).

17. A.L. Fetter, J.D. Walecka, Quantum Theory of ManyParticle Systems (McGraw-Hill, New York, 1971).

18. L.G. Cao, U. Lombardo, C.W. Shen, N. Van Giai, Phys. Rev. C 73, 014313 (2006).

19. B.K. Agrawal, S. Shlomo, V.K. Au, Phys. Rev. C 68, 031304(R) (2005).

20. H. Sagawa S. Yoshida, G.-M. Zeng, J.-Z. Gu, X.-Z. Zhang, Phys. Rev. C 76, 034327 (2007).

21. J. Margueron, P. Chomaz, Phys. Rev. C 67, 041602 (2003).

22. T. Li et al., Phys. Rev. Lett. 99, 162503 (2007).

23. T. Li et al., Phys. Rev. C 81, 034309 (2010).

24. D. Patel et al., Phys. Lett. B 726, 178 (2013) and U. Garg, COMEX3 conference, Mackinac Island (2009).

25. C. Monrozeau et al., Phys. Rev. Lett. 100, 042501 (2008). 